\title{
THERMAL ANALYSIS OF POWER TRANSFORMER
}

Kravchenko E.V.a, Ivleva D.Yu.

Tomsk polytechnic university, 634040, Lenina av.40,Tomsk, Russia

\begin{abstract}
A new approach for predicting the reliability indices based on the numerical analysis of nonuniform temperature fields of power transformer is reported. The failure rates of a power transformer in a real thermal mode of device under natural convection were compared with statistical data.. The necessity of unsteady temperature field consideration was shown to enchance the reliability prediction
\end{abstract}

\section{INTRODUCTION}

Power transformers are used in equipment from several to tens of megawatts. However, regardless of the transformer destination (termination, power, measurement) and power, $80 \%$ of failures occur as a result of coil insulation breakdown [1]. One of the main factors in this kind of damage is the heat destruction of the transformer windings during normal operation [2]. It was established [3] that $10 \mathrm{~K}$ increasing the ambient temperature in working temperature range decreases the lifetime average by $2.5 \%$.. The effect of reducing the operational reliability with increasing temperature (T) is observed not only on the transformers, but for example, when operating the power semiconductor devices [4]. On the other hand the temperature fluctuationscan reach 20-30 K [5], which also leads to increased failure rates (decrease of life) of electrical devices. Therefore, the analysis of thermal modes is necessary to enhance the reliability.Reliability prediction of power transformers is performed using probability modeling [6], including the use of models based on Markov processes [7]. But these methods have significant limitations. This is due not only to a possible lack of information on modes and operating conditions of power transformers, but also with the specification of sample products for testing. The assumption of steady-state operation of the devices and the operating conditions are not always justified.

The aim of this work is mathematical modeling of unsteady inhomogeneous temperature fields of power transformer under natural convection in the presence of a local source of heat to increase the device operational reliability forecast.

\section{THE THERMAL REGIME OF POWER TRANSFORMER ANALYSIS}

The analysis of the thermal mode was based on typical power transformer used in AC-DC inverters within 5 to 50 watts. Solution domain is shown in Figure 1

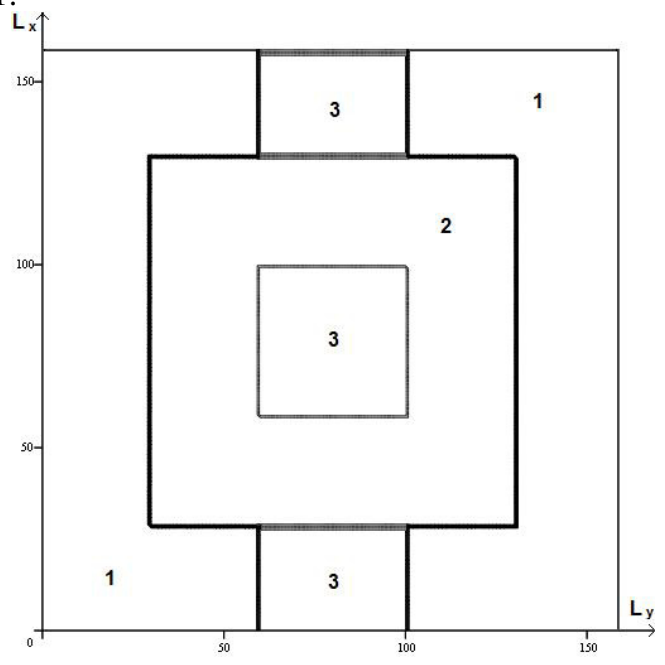

Figure 1. Geometry of the solutions domains (1-3 - regions with different thermal characteristics).

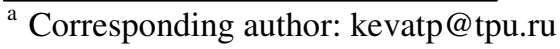


Numerical simulation of temperature field were performed for an inhomogeneous plate with dimensions in the $\mathrm{x}$ and $\mathrm{y}$ equal $L_{X}$ and $L_{Y}$.

$$
x \in\left[0 ; L_{x}\right], y \in\left[0 ; L_{y}\right]
$$

Where: $\mathrm{x}$ and $\mathrm{y}$ is coordinated.

It was assumed that the plate includes areas with different thermal characteristics (Table 1). A local heat intensity $Q$ is assumed for region 2 (Fig. 1.). Convection boundary conditions on the edges of the plate are assumed.

Key assumptions used in the formulation of the problem.

1. Thermophysical properties of materials are independent from a temperature.

2. Ideal thermal contact at the boundaries between the regions is assumed.

Table 1. Thermal characteristics of the of object

\begin{tabular}{|l|l|l|l|}
\hline \multicolumn{1}{|c|}{ Area } & \multicolumn{1}{|c|}{ Density $\rho, \mathrm{kg} / \mathrm{m}^{3}$} & Specific heat $C, \mathrm{~J} /(\mathrm{kg} \cdot \mathrm{K})$ & Thermal conductivity $\lambda$, Watt/(m.K) \\
\hline 1 & 1200 & 1000 & 0,3 \\
\hline 2 & 5000 & 334 & 200 \\
\hline 3 & 4000 & 700 & 4 \\
\hline
\end{tabular}

In this formulation, the problem is reduced to the solution of two-dimensional unsteady heat conduction equation:

$$
\begin{aligned}
& C(x, y) \rho(x, y) \frac{\partial T}{\partial t}=\frac{\partial}{\partial x}\left(\lambda(x, y) \frac{\partial T}{\partial x}\right)+\frac{\partial}{\partial y}\left(\lambda(x, y) \frac{\partial T}{\partial y}\right) \\
& +\frac{Q(t, x, y)}{S h}+\frac{\alpha(T)(T e x t-T)}{h}
\end{aligned}
$$

Where: C-heat capacity; $\rho$ - density; T-temperature; t time; $\lambda$ - thermal conductivity coefficient; $\mathrm{Q}$ - heat source; Ssource area; $h$ - plate thickness; $\alpha$ - convective heat transfer coefficient of the surface; Text- environment temperature.

The coefficient of convective heat transfer is temperature-dependent and determined for every point of the surface.

$$
\alpha(T)=\left(1.42-1.4 \cdot 10^{-3} T_{\text {avr }}\right) N\left(\frac{T-T_{\text {ext }}}{L}\right)^{\frac{1}{4}}
$$

The thermal conductivity equation (2) with appropriate initial and boundary conditions is solved by the finite difference method by analogy with [4,5]. The diagram of splitting by coordinates was applied to the solution of difference analogues of a three-dimensional equation.

\section{STATEMENT OF WORK OF POWER TRANSFORMER RELIABILITY INDICES PREDICTION}

Two mathematical models such as Arrhenius [8] and a multiplicative model [1] were selected for a power transformer reliability analysis. The mathematical multiplicative model [1] of the reliability evaluation diode module is shown below

$$
\lambda_{m}=\lambda_{g} \cdot \mathrm{K}_{T} \cdot \mathrm{K}_{g} \cdot \mathrm{K}_{y_{r}}
$$

where: $\lambda b$-is a base failure rate of the power unit; KT - temperature dependent mode coefficient; Ke - stiffness conditions coefficient; Kpr - quality level coefficient.

It's important to note, that a base failure rate of the power unit $(\lambda \mathrm{b})$ calculated for condition of Tconst=298 $\mathrm{K}$.

It is important to note that the basic failure rate used in mathematical models of the type (3) in determining the reliability indices of power transformer is given for the temperature of $298 \mathrm{~K} \mathrm{[1]} \mathrm{and} \mathrm{do} \mathrm{not} \mathrm{include} \mathrm{the} \mathrm{spatial}$ inhomogeneity of temperature fields inside and outside the unit.

According to [1], a transformers sudden failures occur. $80 \%$ of the failures are due to short-circuit, and $20 \%$ due to an open transformer windings. Short circuit (as a kind of failure) is associated primarily with the physical and chemical processes of degradation (aging) of the windings of the device. Well known that the aging rate (accumulation of degrading conditions) depends not only on the electrical load regimes, but also on temperature conditions of an operation and a storage. Therefore, the analysis of reliability indices of a typical power electronics device (power transformer) is also carried out on the basis of a mathematical model for which $\mathrm{T}$ is one of the most important factors [8].

According to the Arrhenius model the failure rate is exponentially dependent on the temperature [2]:

$$
\lambda_{\mathrm{A}}(T)=C \cdot \exp \left(\frac{-E}{k T}\right)
$$

Where: C-constant, E-activation energy, k-Boltzmann constant.

The temperature used according to (4), was determined from the results of solving two-dimensional unsteady heat conduction equation (2) with appropriate boundary conditions.

Analysis of indicators of reliability (failure rate) of the power transformer (model (4) and (5)) was carried out for steady-state operation of the device and typical operating conditions at mbient temperature ranged from 298 to $318 \mathrm{~K}$. 


\section{RESULTS AND DISCUSSION}

The characteristic form of the temperature field of the power transformer at ambient temperature Text $=298 \mathrm{~K}$ at time $\mathrm{t}=$ $2 \mathrm{~s}$ is shown in Figure 2.
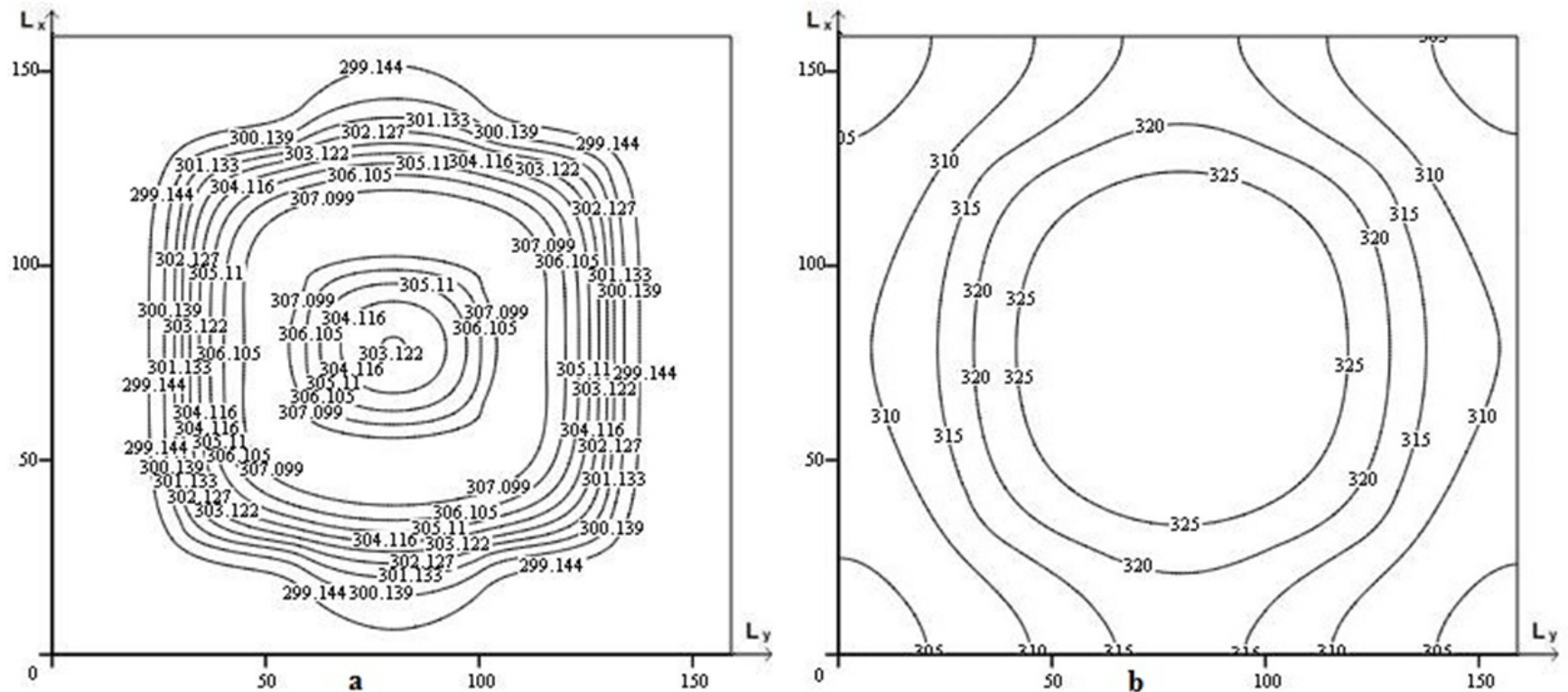

Figure 2. Temperature field of the modeled object $(t=2 s)(a)$ and $t=30 s(b)$.

As represented in Figure 2 the temperature field of the simulated object is typical for giving initial conditions and the mode of operation. Isotherm describes the geometry of the power transformer quite accurately.

Analysis of the nature of heat distribution shows that the most high temperature region is in the center. Temperature field of the device under consideration is significantly heterogeneous and characterized by significant gradients of $\mathrm{T}$. Therefore, the reliability of forecasting of power transformer using the Arrhenius model is advisable for the average (Tav) and maximum (Tmax) temperatures of the object.

The results of numerical modeling of reliability indices (failure rate) power transformer are shown in Fig. 3. Failure rate behavior $\lambda \mathrm{A}(\mathrm{T})$ shows not only significant differences in the estimates of reliability models (4) and (5), but also a high degree of dependence of the Arrhenius predictive model (curves 1, 2 and 3 in Figure 3) calculated from (received) temperature.
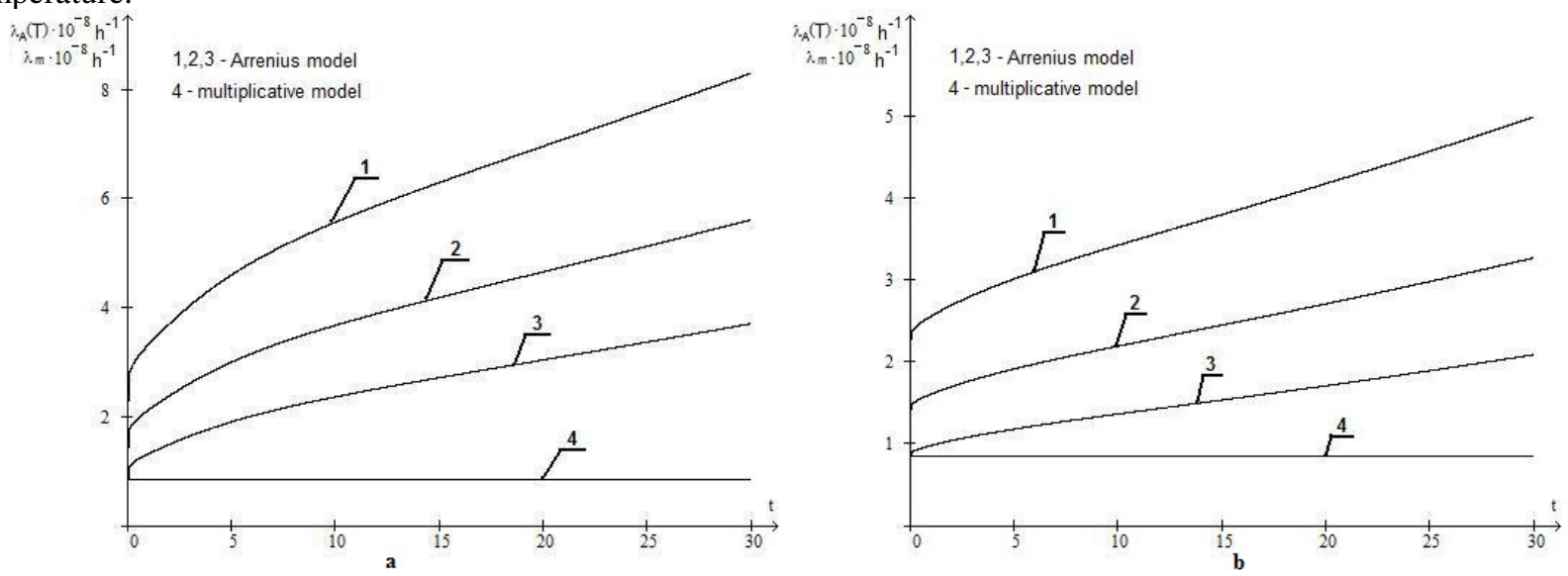

Figure 3. The failure rate of the power transformer designed for maximum temperature(a) and average temperature (b) modeled object: 1 - Text $=298 \mathrm{~K} ; 2-$ Text $=308 \mathrm{~K} ; 3-$ Text $=318 \mathrm{~K}$.

Dependency analysis presented in Figure $3 \mathrm{a}$ shows that the numerical values of $\lambda \mathrm{A}(\mathrm{Tmax})$ calculated from the Arrhenius model (5), 4, 6 and 10 times higher than those obtained by the multiplicative model (4) for the time of 30s and ambient temperatures 298,308 and $318 \mathrm{~K}$.

It is obvious that with increasing $\mathrm{T}$ the operating temperature range of transformer (e.g., in an objective increase in ambient temperature), the failure rate of these devices must grow or, in other words, the values of reliability indices should decrease. The latter should affect, for example, the service life of equipment and power electronics for the reliability of the system containing these transformerts. 
Analysis of the failure rates (Figure $3 b$ ) shows that the numerical values $\lambda A($ Tav) calculated from the Arrhenius model (4), at 2, 4 and 6 times greater than in the model (3) for the time of 30s at ambient temperatures 298,308 and 318 $\mathrm{K}$. The intensity ratio of failures Arrhenius model $\lambda \mathrm{A}(\mathrm{Tmax})$ for $\lambda \mathrm{A}(\mathrm{Tav})$ was 1.6 in simulations Text $=318 \mathrm{~K}$.

Significant differences in the estimates of reliability are due to several reasons:

- $\quad$ significant variation of the temperature field of the simulated power electronics devices (due to local sources of heat);

- $\quad$ base failure rate $\left(\lambda_{\mathrm{b}}\right.$ in equation 3$)$ used in the evaluation $\lambda_{\mathrm{m}}$, defined for $\mathrm{T}=298 \mathrm{~K}$;

- The intensity of convective heat transfer reduction occurs with increasing $\mathrm{T}_{\text {ext }}$, causing even greater heterogeneity of temperature fields with a corresponding increase of the maximum and average temperatures for the device.

The proposed mathematical tool for the analysis of thermal conditions can be used as a the basis for POF (POF Physic of Failure) methodes. Utilization of the methods in predicting electrical devices POF reliability allows minimizing the number of acceptance tests. Thus the methods based on the physics of failure [9-14], may be the important component concepts of PDfR (PDfR - Probabilistic Design for Reliability) [15] and DRM (DRM - Dynamic Reliability Management) [16].

\section{Conclusion}

Using the multiplicative model (3) to estimate the failure rate of power transformers results in a significant overestimation of the devices operating lifetime.

A power transformer reliability forecast should be carried out based on the analysis of real inhomogeneous unsteady thermal mode of the device.

The work was supported by the RFFI (project № 15-38-50595).

\section{References}

[1] S.F. Prytkov, V.M. Gorbachev, Handbook «Reliability of radioelements», Moscow, (2012)

[2] A.O. Valuyskih, I.N. Dulkin, A.A. Filippov, G.M. Tsfasman, Modeling of the thermal regime of the transformer in the control, monitoring and diagnostics, Electro, № 1. pp. 15-19, (2008)

[3] A.N. Nazarychev, D.A, Andreev, Pedro Antonio, E.A. Kireev, Investigation of the influence of ambient temperature on the electrical consumption of the resource, Bull of the Ivanovo State Power University, № 3. pp. 57-60, (2009)

[4] G.V. Kuznetsov, E.V. Kravchenko, The peculiarities of modeling reliability parameters for printed circuit assembly electronics working in cycling mode, Elektromagnitnye Volny i Elektronnye Systemy, № 10 (11-12), pp. 19-22, (2005)

[5] G.V. Kuznetsov, M.A. Sheremet, New approach to the mathematical modeling of thermal regimes for electronic equipment, Russian Microelectronics, № 2, pp. 131-138, (2008)

[6] V.M. Saltykov, L.M. Suleimanova, Predicting the service life of power transformers of electrical networks using probabilistic modeling, News of higher educational institutions. Electromechanics, № 6, pp. 65-67, (2007)

[7] M.Sefidgaran, M. Mirzaie, A. Ebrahimzadeh, Reliability model of the power transformer with ONAF cooling, Int. J. Electr. Power Energy Syst., V.35, Issue 1, pp. 97-104, (2012), doi: 10.1016/j.ijepes.2011.10.002

[8] A.A. Borisov, V.M. Gorbachev, G.D. Kartashow, M.N. Martynov, S.F. Prytkov, Reliability foreign element base, Foreign electronics, № 5. pp. 34-53, (2000)

[9] G.V. Kuznetsov, E.V. Kravchenko, Influence of polymer aging on reliability indices of a typical printed-circuit assembly of radioelectronic equipment, Journal of Engineering Physics and Thermophysics, vol. 80, №5, pp. 10501054, (2007), doi: 10.1007/s10891-007-0137-2

[10] G.V. Kuznetsov, E.V. Kravchenko, Prediction of power semiconductors devices reliability working in cyclic mode, EPJ Web of Conferences, 76, 01014, (2014), doi: 10.1051/epjconf/20147601014

[11] G.V. Kuznetsov, E.V. Kravchenko, A new approach to numerical analysis of reliability indices in electronics. EPJ Web Conf., 82, (2015), doi: 10.1051/epjconf/20158201029

[12] G.V. Kuznetsov, E.V. Kravchenko, Increase resource power electronics module on the physics of failure method. MATEC Web Conf., 19, (2014), doi: 10.1051/matecconf/20141901028

[13] E.V. Kravchenko, D.Yu. Ivleva, The reliability of the power semiconductor module on the operating temperature, MATEC Web Conf., 19, (2014), doi: 10.1051/matecconf/20141901002

[14] Kurmangaliev R, Kravchenko E, Research methods of reliability indicators of rectifier diode in tablet execution. EPJ Web Conf., 82, (2015), doi: 10.1051/epjconf/20158201030

[15] E.Suhir, Could electronics reliability be predicted, quantified and assured?, Microelectron Reliab, 53, (2013), pp.925-936, doi: 10.1016/j.microrel.2013.03.011.

[16] Wang Y, Enachescu M, Cotofana SD, Fang L (2012) Variation tolerant on-chip degradation sensors for dynamic reliability management systems. Microelectron Reliab 52:1787-1791. doi: 10.1016/j.microrel.2012.06.038 